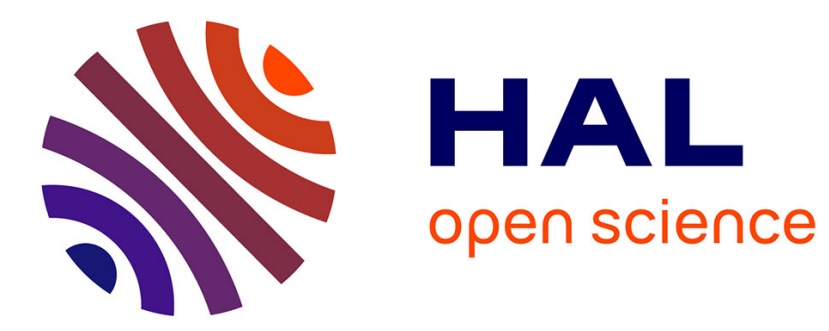

\title{
Lower compactness estimates for scalar balance laws
}

Fabio Ancona, Olivier Glass, Khai Tien Nguyen

\section{To cite this version:}

Fabio Ancona, Olivier Glass, Khai Tien Nguyen. Lower compactness estimates for scalar balance laws.

Communications on Pure and Applied Mathematics, 2012, 65 (9), pp.1303-1329. hal-00606311v2

\section{HAL Id: hal-00606311 \\ https://hal.science/hal-00606311v2}

Submitted on 28 Oct 2011

HAL is a multi-disciplinary open access archive for the deposit and dissemination of scientific research documents, whether they are published or not. The documents may come from teaching and research institutions in France or abroad, or from public or private research centers.
L'archive ouverte pluridisciplinaire HAL, est destinée au dépôt et à la diffusion de documents scientifiques de niveau recherche, publiés ou non, émanant des établissements d'enseignement et de recherche français ou étrangers, des laboratoires publics ou privés. 


\title{
Lower compactness estimates for scalar balance laws
}

\author{
Fabio Ancona*, Olivier Glass ${ }^{\dagger}$, Khai T. Nguyen* \\ Dedicated to Prof. Constantine Dafermos in the occasion of his $70^{\text {th }}$ birthday
}

October 11, 2011

\begin{abstract}
In this paper, we study the compactness in $L_{l o c}^{1}$ of the semigroup $\left(S_{t}\right)_{t \geq 0}$ of entropy weak solutions to strictly convex scalar conservation laws in one space dimension. The compactness of $S_{t}$ for each $t>0$ was established by P. D. Lax [10]. Upper estimates for the Kolmogorov's $\varepsilon$-entropy of the image through $S_{t}$ of bounded sets in $L^{1} \cap L^{\infty}$ were given by C. De Lellis and F. Golse [5]. Here, we provide lower estimates on this $\varepsilon$-entropy of the same order as the one established in [5], thus showing that such an $\varepsilon$-entropy is of size $\approx(1 / \varepsilon)$. Moreover, we extend these estimates of compactness to the case of convex balance laws.
\end{abstract}

\section{Introduction}

Consider a scalar conservation law in one space dimension

$$
u_{t}+f(u)_{x}=0,
$$

where $u=u(t, x)$ is the state variable, and $f: \mathbb{R} \rightarrow \mathbb{R}$ is a twice continuously differentiable, (uniformly) strictly convex function:

$$
f^{\prime \prime}(u) \geq c>0 \quad \forall u \in \mathbb{R} .
$$

Without loss of generality, we will suppose

$$
f^{\prime}(0)=0
$$

since one may always reduce the general case to this one by performing the space-variable and flux transformations $x \rightarrow x+t f^{\prime}(0)$ and $f(u) \rightarrow f(u)-u f^{\prime}(0)$. We recall that problems of this type do not possess classical solutions since discontinuities arise in finite time even if the initial data are smooth. Hence, it is natural to consider weak solutions in the sense of distributions that, for sake of uniqueness, satisfy an entropy criterion for admissibility [4]:

$$
u(t, x-) \geq u(t, x+) \quad \text { for a.e. } t>0, \quad \forall x \in \mathbb{R},
$$

where $u(t, x \pm)$ denote the one-sided limits of $u(t, \cdot)$ at $x$. The equation (1) generates an $L^{1}$-contractive semigroup of solutions $\left(S_{t}\right)_{t \geq 0}$ that associates, to every given initial data $u_{0} \in L^{1}(\mathbb{R}) \cap L^{\infty}(\mathbb{R})$, the unique entropy admissible weak solution $S_{t} u_{0} \doteq u(t, \cdot)$ of the corresponding Cauchy problem $(\mathrm{cfr}$. [4, 9]). This yields the existence of a continuous semigroup $\left(S_{t}\right)_{t \geq 0}$ acting on the whole space $L^{1}(\mathbb{R})$. Such a semigroup $S_{t}$ was shown by Lax [10] to be compact as a mapping from $L^{1}(\mathbb{R})$ to $L_{\text {loc }}^{1}(\mathbb{R})$, for every $t>0$. De Lellis and Golse [5], following a suggestion by Lax [10], used the Kolmogorov's $\varepsilon$-entropy concept, which is recalled below, to provide a quantitative version of this compactness effect.

\footnotetext{
*Dipartimento di Matematica Pura ed Applicata, Università degli Studi di Padova, Via Trieste 63, 35121 Padova, Italy

†Ceremade, Université Paris-Dauphine, CNRS UMR 7534, Place du Maréchal de Lattre de Tassigny, 75775 Paris Cedex 16, France
} 
Definition 1. Let $(X, d)$ be a metric space and $K$ a totally bounded subset of $X$. For $\varepsilon>0$, let $N_{\varepsilon}(K)$ be the minimal number of sets in a cover of $K$ by subsets of $X$ having diameter no larger than $2 \varepsilon$. Then the $\varepsilon$-entropy of $K$ is defined as

$$
H_{\varepsilon}(K \mid X) \doteq \log _{2} N_{\varepsilon}(K)
$$

Throughout the paper, we will call an $\varepsilon$-cover, a cover of $K$ by subsets of $X$ having diameter no larger than $2 \varepsilon$.

De Lellis and Golse obtained an upper bound for the $\varepsilon$-entropy of the set of solutions to (1) at any given time $t>0$, as $\varepsilon \rightarrow 0^{+}$; that is to say, they showed how strong is the compactifying effect. Precisely, they established the following result.

Theorem $1([5])$. Consider a twice continuously differentiable $f: \mathbb{R} \rightarrow \mathbb{R}$, satisfying (2), (3). Given any $L, m, M>0$, define the set of bounded, compactly supported initial data

$$
\mathcal{C}_{[L, m, M]} \doteq\left\{u_{0} \in L^{1}(\mathbb{R}) \cap L^{\infty}(\mathbb{R}) \mid \operatorname{Supp}\left(u_{0}\right) \subset[-L, L],\left\|u_{0}\right\|_{L^{1}} \leq m,\left\|u_{0}\right\|_{L^{\infty}} \leq M\right\} .
$$

Then for $\varepsilon>0$ sufficiently small, one has

$$
H_{\varepsilon}\left(S_{T}\left(\mathcal{C}_{[L, m, M]}\right) \mid L^{1}(\mathbb{R})\right) \leq \frac{4}{\varepsilon}\left(\frac{4 L(T)^{2}}{c T}+4 L(T) \sqrt{\frac{2 m}{c T}}\right) \quad \forall T>0,
$$

with

$$
L(T) \doteq L+2 \sup _{|z| \leq M}\left|f^{\prime \prime}(z)\right| \sqrt{2 m T / c} .
$$

The aim of this paper is to show that the $\varepsilon$-entropy estimates provided by Theorem 1 turn out to be optimal, since we shall establish a lower bound on such an $\varepsilon$-entropy which is of the same order as De Lellis and Golse's upper bounds. Hence, we deduce that $H_{\varepsilon}\left(S_{T}\left(\mathcal{C}_{[L, m, M]}\right) \mid L^{1}(\mathbb{R})\right)$ is exactly of size $\approx \varepsilon^{-1}$. Precisely, we prove the following.

Theorem 2. Under the assumptions and in the same setting of Theorem 1, for any $T>0$, and for $\varepsilon>0$ sufficiently small, one has

$$
H_{\varepsilon}\left(S_{T}\left(\mathcal{C}_{[L, m, M]}\right) \mid L^{1}(\mathbb{R})\right) \geq \frac{1}{\varepsilon} \cdot \frac{L^{2}}{48 \cdot \ln (2) \cdot\left|f^{\prime \prime}(0)\right| T} .
$$

As suggested in [10], the knowledge of the $\varepsilon$-entropy magnitude of the solution set of (1) may play an important role to provide estimates on the accuracy and resolution of numerical methods for (1).

The main steps of the proof of the lower bound (8) consist in:

1. Introducing a suitable class of piecewise affine functions and showing that any element of such a class can be obtained, at any given time $t$, as the value $u(t, \cdot)$ of an entropy admissible weak solution of (1), with initial data in $\mathcal{C}_{[L, m, M]}$.

2. Providing an optimal estimate of the maximum number of functions in such a class that can be contained in a subset of $S_{T}\left(\mathcal{C}_{[L, m, M]}\right)$ having diameter no larger than $2 \varepsilon$. This estimate is established with a similar combinatorial argument as the one used in [1].

Remark 1. Since by (2), (7), we have $L(T) \sqrt{\frac{2 m}{c T}} \leq \frac{L(T)^{2}}{2 c T}$, one derives from (6) the estimate

$$
H_{\varepsilon}\left(S_{T}\left(\mathcal{C}_{[L, m, M]}\right) \mid L^{1}(\mathbb{R})\right) \leq \frac{1}{\varepsilon} \cdot \frac{24 L(T)^{2}}{c T}
$$

Therefore, the size $\frac{1}{\varepsilon} \cdot \frac{L^{2}}{\left|f^{\prime \prime}(0)\right| T}$ of the lower bound (8) turns out to be the same as the one of the upper bound on the $\varepsilon$-entropy of $S_{T}\left(\mathcal{C}_{[L, m, M]}\right)$ provided by Theorem 1, upon replacing $L$ with $L(T)$, and $\left|f^{\prime \prime}(0)\right|$ with $c$. 
Next, we address the more general case of convex balance laws. Namely, given a twice continuously differentiable map $f: \mathbb{R} \rightarrow \mathbb{R}$ satisfying (2), (3), we will analyze the compactifying effect of the balance law

$$
u_{t}+f(u)_{x}=g(t, x, u)
$$

As for (1) we will consider weak solutions of (9) that satisfy the entropy admissibility condition (4). The source term is assumed to be a continuously differentiable map $g: \mathbb{R}^{+} \times \mathbb{R} \times \mathbb{R} \rightarrow \mathbb{R}$, that satisfies the following assumptions:

$$
\begin{aligned}
& g(t, x, 0)=0 \quad \forall(t, x) \in \mathbb{R}^{+} \times \mathbb{R}, \\
& \exists C>0 \text { s.t. }\left|g_{x}(t, x, u)\right| \leq C|u| \quad \forall(t, x, u) \in \mathbb{R}^{+} \times \mathbb{R} \times \mathbb{R}, \\
& \exists \omega \in L_{l o c}^{1}\left(\mathbb{R}^{+}\right) \text {s.t. }\left|g_{u}(t, x, u)\right| \leq \omega(t) \quad \text { for a.e. } t \in \mathbb{R}^{+}, \forall(x, u) \in \mathbb{R}^{2} \text {. }
\end{aligned}
$$

In particular, (10), (12) together imply

$$
\exists \omega \in L_{l o c}^{1}\left(\mathbb{R}^{+}\right) \text {s.t. }|g(t, x, u)| \leq \omega(t) \cdot|u| \quad \text { for a.e. } t \in \mathbb{R}^{+}, \forall(x, u) \in \mathbb{R}^{2} .
$$

Under assumptions (12) or (13), for each $u_{0} \in L^{1}(\mathbb{R}) \cap L^{\infty}(\mathbb{R})$, there exists a unique entropy admissible solution $u(t, x)$ of $(9)$ with initial condition $u(0, \cdot)=u_{0}$, see $[4,7,9]$.

Remark 2. Condition (10) in particular implies the fact that the source term $g$, if not zero, does depend on $u$, since otherwise one would have $g=g(t, x)=0$, for all $t, x$. Because of $(10)$, all solutions $u(t, \cdot)$ to (9) with initial data of compact support remain compactly supported for all times $t>0$.

We shall denote by $E_{t}$ the evolution operator that associates, to every initial data $u_{0} \in L^{1}(\mathbb{R}) \cap L^{\infty}(\mathbb{R})$, the entropy admissible solution $E_{t} u_{0} \doteq u(t, \cdot)$ of the corresponding Cauchy problem for (9). We establish the following.

Theorem 3. Let $f: \mathbb{R} \rightarrow \mathbb{R}$ be a twice continuously differentiable map that satisfies (2), (3), and $g: \mathbb{R}^{+} \times \mathbb{R} \times \mathbb{R} \rightarrow \mathbb{R}$ be a continuously differentiable map that satisfies (10), (11), (12). Then, in the same setting of Theorem 1, for any $T>0$ and for $\varepsilon>0$ sufficiently small, one has

$$
H_{\varepsilon}\left(E_{T}\left(\mathcal{C}_{[L, m, M]}\right) \mid L^{1}(\mathbb{R})\right) \geq \frac{1}{\varepsilon} \cdot \frac{L^{2} \cdot \exp \left(-\|\omega\|_{L^{1}(0, T)}\right)}{48 \cdot \ln (2) \cdot\left|f^{\prime \prime}(0)\right| T} .
$$

Since balance laws are not considered in [5], following the same lines of the proof in [5] we also establish the same type of upper bound for $H_{\varepsilon}\left(E_{T}\left(\mathcal{C}_{[L, m, M]}\right) \mid L^{1}(\mathbb{R})\right)$ as the one given in Theorem 1.

Let us introduce the following notations. Given $t>0, M>0$ and $a, b \in \mathbb{R}$ with $a<b$, we set

$$
\Delta_{a, b, t}(M) \doteq\left\{(s, x) \mid s \in[0, t], \quad a-(t-s) \cdot\left\|f^{\prime}\right\|_{L^{\infty}(-M, M)} \leq x \leq b+(t-s) \cdot\left\|f^{\prime}\right\|_{L^{\infty}(-M, M)}\right\},
$$

and

$$
k_{a, b, t}(M) \doteq \max \left\{\left|g_{x}(s, x, u)\right| ;(s, x) \in \Delta_{a, b, t}(M), u \in[-M, M]\right\} .
$$

We obtain the following result.

Theorem 4. In the same setting of Theorem 1 , assume that $f: \mathbb{R} \rightarrow \mathbb{R}$ is a twice continuously differentiable map that satisfies (2), (3), and $g: \mathbb{R}^{+} \times \mathbb{R} \times \mathbb{R} \rightarrow \mathbb{R}$ is a continuously differentiable map that satisfies (10), (12). Then, for $\varepsilon>0$ sufficiently small, one has

$$
H_{\varepsilon}\left(E_{T}\left(\mathcal{C}_{[L, m, M]}\right) \mid L^{1}(\mathbb{R})\right) \leq \frac{1}{\varepsilon} \cdot \frac{8 L(T)^{2} \cdot\left(1+2\left(1+c T^{2} K_{L, T}\right) \exp \left(\|\omega\|_{L^{1}(0, T)}\right)\right)}{c T} \quad \forall T>0,
$$

where

$$
\begin{gathered}
L(T) \doteq L+2\left\|f^{\prime \prime}\right\|_{L^{\infty}\left(-M_{T}, M_{T}\right)} \sqrt{\frac{2 m T}{c}}\left[1+T \sqrt{c K_{L, T}}\right] \cdot \exp \left(\|\omega\|_{L^{1}(0, T)}\right), \\
K_{L, T} \doteq k_{-L_{T}, L_{T}, T}\left(M_{T}\right)
\end{gathered}
$$


with

$$
L_{T} \doteq L+\left\|f^{\prime \prime}\right\|_{L^{\infty}\left(-M_{T}, M_{T}\right)} \cdot M_{T} T
$$

and

$$
M_{T} \doteq \exp \left(\|\omega\|_{L^{1}(0, T)}\right) \cdot M
$$

Remark 3. Theorems 3 and 4 remain true if the source term has the form $g=g(t, u)$, and satisfies only the condition (12), together with $g(\cdot, 0) \in L_{l o c}^{1}$. Clearly, in this case, the solution $u(t, \cdot)$ of $(9)$ will not be in general compactly supported, but instead the difference between $u(t, \cdot)$ and the solution of (9) with zero initial data has always compact support. So, it will be convenient to compute the $\varepsilon$-entropy of the translated set $E_{T}\left(\mathcal{C}_{[L, m, M]}\right)-E_{T} 0$, which obviously coincides with the one of $E_{T}\left(\mathcal{C}_{[L, m, M]}\right)$. In this way we will see in Subsection 4.3 that one can establish, for $\varepsilon>0$ sufficiently small, the estimate

$$
H_{\varepsilon}\left(E_{T}\left(\mathcal{C}_{[L, m, M]}\right) \mid L^{1}(\mathbb{R})\right) \leq \frac{1}{\varepsilon} \cdot \frac{8 L(T)^{2} \cdot\left(1+2 \exp \left(\|\omega\|_{L^{1}(0, T)}\right)\right)}{c T} \quad \forall T>0,
$$

where

$$
L(T) \doteq L+2\left\|f^{\prime \prime}\right\|_{L^{\infty}\left(-M_{T}^{g}, M_{T}^{g}\right)} \sqrt{\frac{2 m T}{c}} \cdot \exp \left(\|\omega\|_{L^{1}(0, T)}\right),
$$

with

$$
M_{T}^{g} \doteq \exp \left(\|\omega\|_{L^{1}(0, T)}\right)\left(M+\|g(\cdot, 0)\|_{L^{1}(0, T)}\right) .
$$

Moreover, for any $T>0$, and for $\varepsilon>0$ sufficiently small, we derive the estimate

$$
H_{\varepsilon}\left(E_{T}\left(\mathcal{C}_{[L, m, M]}\right) \mid L^{1}(\mathbb{R})\right) \geq \frac{1}{\varepsilon} \cdot \frac{L^{2} \cdot \exp \left(-\|\omega\|_{L^{1}(0, T)}\right)}{24 \cdot \ln (2) \cdot\left\|f^{\prime \prime}\right\|_{L^{\infty}\left(-G_{T}, G_{T}\right)} T} .
$$

where

$$
G_{T} \doteq 1+\|g(\cdot, 0)\|_{L^{1}(0, T)} \exp \left(\|\omega\|_{L^{1}(0, T)}\right) .
$$

As a final remark, we observe that it would be interesting to provide upper and lower quantitative compactness estimates for the solution set of genuinely nonlinear $2 \times 2$ systems of conservation laws (whose $L_{l o c}^{1}$ compactness follows from the estimates provided in [6], as observed in [11]), while it remains a completely open problem whether such a compactness property continues to hold (and possibly derive similar quantitative estimates) for general systems of $N$ conservation laws with genuinely nonlinear characteristic fields.

The paper is organized as follows. In Section 2 we provide a tight lower bound for the $\varepsilon$-entropy of the solution set of a convex conservation law, establishing Theorem 2. In Section 3 we derive an Oleinik type inequality for convex balance laws with smooth source term, and then extend the results of Section 2 to the case of convex balance laws, proving Theorem 3. Finally, in Section 4 we derive an upper bound for the $\varepsilon$-entropy of the solution set of a convex balance law, proving Theorem 4; also, we prove Remark 3.

Acknowledgements. The authors would like to warmly thank the anonymous referee for a comment and a suggestion that has contributed to simplify some proofs and to obtain slightly more general results for balance laws. They wish to thank Institut Henri Poincaré (Paris, France) for providing a very stimulating environment at the "Control of Partial Differential Equations and Applications" program in the Fall 2010, during which a part of this work was written. FA and KTN are partially supported by the European Union Seventh Framework Programme [FP7-PEOPLE-2010-ITN] under grant agreement n.264735-SADCO. OG is partially supported by the Agence Nationale de la Recherche, Project CISIFS, grant ANR-09-BLAN-0213-02.

\section{Lower compactness estimates for conservation laws}

\section{$2.1 \quad$ Proof of Theorem 2}

For arbitrary positive constants $L, M, m$ and $b$, let us consider the set

$$
\mathcal{A}_{[L, m, M, b]} \doteq\left\{u_{T} \in \mathrm{BV}(\mathbb{R}) \mid \operatorname{Supp}\left(u_{T}\right) \subset[-L, L],\left\|u_{T}\right\|_{L^{1}} \leq m,\left\|u_{T}\right\|_{L^{\infty}} \leq M, D u_{T} \leq b\right\},
$$


where the last inequality has to be understood in the sense of measures, i.e. the Radon measure $D u_{T}$ satisfies $D u_{T}(J) \leq b \cdot|J|$ for every Borel set $J \subset \mathbb{R},|J|$ being the Lebesgue measure of $J$. We obtain a proof of Theorem 2 as a consequence of the following two propositions that shall be established below.

Proposition 1. Suppose that $f: \mathbb{R} \rightarrow \mathbb{R}$ is a twice continuously differentiable map satisfying (2), (3). Then, given any $L, m, M, T>0$, for

$$
0 \leq h \leq \min \left(M, \frac{m}{2 L}, \frac{L}{8 T\left|f^{\prime \prime}(0)\right|}\right),
$$

sufficiently small, one has

$$
\mathcal{A}_{\left[L_{T}, L h, h,\left(2 T\left|f^{\prime \prime}(0)\right|\right)^{-1}\right]} \subset S_{T}\left(\mathcal{C}_{[L, m, M]}\right),
$$

with

$$
L_{T} \doteq L-2 T\left|f^{\prime \prime}(0)\right| \cdot h .
$$

Proposition 2. Given $L, m, M, b>0$, for any $\varepsilon>0$ satisfying

$$
\varepsilon \leq \frac{\min (m, L M)}{6},
$$

one has

$$
H_{\varepsilon}\left(\mathcal{A}_{[L, m, M, b]} \mid L^{1}(\mathbb{R})\right) \geq \frac{1}{\varepsilon} \cdot \frac{2 b L^{2}}{27 \ln (2)} .
$$

Notice that the lower bound (24) is independent on $m$ and $M$, which appear only in the constraint (23). Moreover, because of (20), the constant $L_{T}$ given by (22) satisfies $L_{T} \geq(3 / 4) L$. Hence, applying (24), with $L=L_{T}, b=\left(2 T\left|f^{\prime \prime}(0)\right|\right)^{-1}$, and relying on (21), we recover the estimate (8), which proves Theorem 2.

\subsection{Proof of Proposition 1}

1. We shall first prove the inclusion

$$
\mathcal{A}_{\left[L_{T}, 2 L h, h,\left(2 T\left|f^{\prime \prime}(0)\right|\right)^{-1}\right]} \cap C^{1}(\mathbb{R}) \subset S_{T}\left(\mathcal{C}_{[L, m, M]}\right),
$$

$\left(C^{1}(\mathbb{R})\right.$ denoting the set of continuously differentiable maps on $\mathbb{R}$ with values in $\left.\mathbb{R}\right)$. More precisely, we will show that any element $u_{T} \in C^{1}(\mathbb{R})$ of the set on the left-hand side of $(21)$ can be obtained as the value at time $T$ of a weak admissible solution to (1), which is backward constructed starting from $u_{T}$ by reversing the direction of time. Namely, given

$$
u_{T} \in \mathcal{A}_{\left[L_{T}, 2 L h, h,\left(2 T\left|f^{\prime \prime}(0)\right|\right)^{-1}\right]} \cap C^{1}(\mathbb{R}),
$$

set

$$
w_{0}(x) \doteq u_{T}(-x) \quad \forall x \in \mathbb{R},
$$

and consider the entropy weak solution $w(t, x) \doteq S_{t} w_{0}$ of (1) with initial data $w_{0}$. By well-known properties of solutions to scalar conservation laws, and because of (20), (26), w verifies the $L^{1}$ and $L^{\infty}$ bounds (cfr. [4, Theorem 6.2.3, Theorem 6.2.6]):

$$
\begin{aligned}
\|w(t, \cdot)\|_{L^{1}} & \leq\left\|w_{0}\right\|_{L^{1}} \leq 2 L h \leq m, \quad \forall t>0 . \\
\|w(t, \cdot)\|_{L^{\infty}} & \leq\left\|w_{0}\right\|_{L^{\infty}} \leq h \leq M,
\end{aligned}
$$

Next, observe that the function

$$
u(t, x) \doteq w(T-t,-x), \quad(t, x) \in[0, T] \times \mathbb{R},
$$

is a weak solution of (1) in the sense of distribution, which, by (27), clearly satisfies

$$
u(T, \cdot)=u_{T} .
$$


On the other hand, if we show that for all $t \in[0, T[, x \mapsto w(t, x)$ is smooth on $\mathbb{R}$, it would follow that $u(t, x)$ automatically satisfies the admissibility condition (4) as an equality and thus provides an entropy weak solution of (1) which attains the value $u_{T}$ at time $T$. To this end, recalling from (26), (27) that $w_{0}(\cdot) \in C^{1}(\mathbb{R})$, by the classical method of characteristics (e.g, see [2, Chapter 3]) one can show that, setting

$$
\left.T_{1} \doteq \sup \left\{\tau \in[0, T] \mid w(t, \cdot) \in C^{1}(\mathbb{R}) \quad \forall t \in[0, \tau]\right)\right\}
$$

there holds

$$
T_{1}<T \quad \Longrightarrow \quad \sup _{a>0} \lim _{t \rightarrow T_{1}-}\left\|w_{x}(t, \cdot)\right\|_{L^{\infty}(-a, a)}=\infty .
$$

Next, observing that the function

$$
v(t, x) \doteq w_{x}(t, x),
$$

is the unique broad solution on $\left[0, T_{1}[\times \mathbb{R}\right.$ of the semilinear equation

$$
v_{t}(t, x)+f^{\prime}(w(t, x)) \cdot v_{x}(t, x)=-f^{\prime \prime}(w(t, x)) \cdot v(t, x)^{2}
$$

(e.g, see [2, Theorems 3.1 and 3.6]), it follows that the value

$$
z(t) \doteq v(t, x(t))
$$

of $v$ along a characteristic $x(\cdot)$ of (1) satisfies the equation

$$
\dot{z}(t)=-f^{\prime \prime}(w(t, x(t))) \cdot z^{2}(t), \quad t \in\left[0, T_{1}[.\right.
$$

As a consequence of $(2),(33)$, we deduce that $z(\cdot)$ is decreasing on $\left[0, T_{1}[\right.$, and thus one has $z(t) \leq z(0)$ for all $t \in\left[0, T_{1}[\right.$. Hence, if we show that

$$
z(t) \geq-2\left(T f^{\prime \prime}(0)\right)^{-1} \quad \forall 0 \leq t<T_{1},
$$

it would follow that $z(t)$ is uniformly bounded on $\left[0, T_{1}\right.$ [ which, in turn, implies the uniform boundedness of $w_{x}(t, x)$ for $t \in\left[0, T_{1}\left[, x \in \mathbb{R}\right.\right.$. Therefore, by (31), to prove that $T=T_{1}$, it suffices to establish the lower bound (34). Notice that by the continuity of $z(\cdot)$, in order to prove (34), it will not be restrictive to treat only the case $z(0)<0$. On the other hand recall that by $(26),(27)$ we have $z(0) \geq-\left(2 T f^{\prime \prime}(0)\right)^{-1}$. Next, observe that by the continuity assumption on $f^{\prime \prime}$, we may assume that

$$
f^{\prime \prime}(h) \leq \frac{3}{2} f^{\prime \prime}(0)
$$

for $|h|$ sufficiently small. Hence, relying on (33), (35), we derive $\dot{z}(t) \geq-\frac{3}{2} f^{\prime \prime}(0) \cdot z^{2}(t)$, which yields

$$
\frac{1}{z(t)} \leq \frac{1}{z(0)}+\frac{3}{2} f^{\prime \prime}(0) t \leq-\frac{f^{\prime \prime}(0) T}{2} \quad \forall 0 \leq t<T_{1} .
$$

Clearly, from (36) we recover the bound (34). Thus, by the above observation we conclude that $T_{1}=T$, and that $u(t, \cdot)=w(T-t,-\cdot) \in C^{1}(\mathbb{R})$ for all $\left.t \in\right] 0, T\left[\right.$. Therefore, to complete the proof of $u_{T} \in$ $S_{T}\left(\mathcal{C}_{[L, m, M]}\right)$, we only need to show that the initial condition $u(0, \cdot)=w(T,-\cdot)$ is an element of the set $\mathcal{C}_{[L, m, M]}$ defined in (5). By the estimates (28) we already know that $u(0, \cdot)$ satisfies the $L^{1}$ and $L^{\infty}$ bounds of $\mathcal{C}_{[L, m, M]}$. On the other hand, observing that the solution $w$ propagates along classical characteristics, and relying on (3), (26), (27), (28), (35), we derive (for $h$ sufficiently small) the bound on the support of $w$ :

$$
\operatorname{Supp}(w(t, \cdot)) \subset[-l(t), l(t)], \quad \forall t \in[0, T],
$$

where $l(t) \doteq L_{T}+\frac{3}{2} t\left|f^{\prime \prime}(0)\right| \cdot h$. In turn, (37) together with (22) yields

$$
\operatorname{Supp}(u(0, \cdot))=\operatorname{Supp}(w(T,-\cdot)) \in[-L, L] .
$$

Hence, $u(0, \cdot) \in \mathcal{C}_{[L, m, M]}$ and the proof of $(25)$ is completed.

2. In order to establish (21), observing that for any $u_{T} \in \mathcal{A}_{\left[L_{T}, L h, h, b_{0}\right]}, b_{0} \doteq\left(2 T\left|f^{\prime \prime}(0)\right|\right)^{-1}$, the map $x \mapsto u_{T}(x)-b_{0} x$ is nonincreasing, we may find a sequence of nonincreasing functions $v_{T}^{n}$ in $C^{1}(\mathbb{R})$, 
such that $v_{T}^{n}(x) \leq u_{T}(x)-b_{0} x$, for all $x \in \mathbb{R}, \lim _{n \rightarrow \infty}\left\|v_{T}^{n}-u_{T}+b_{0} \cdot\right\|_{L^{1}}=0$, and $\left\|v_{T}^{n}+b_{0} \cdot\right\|_{L^{1}} \leq 2 L h$. Hence, for any given $u_{T} \in \mathcal{A}_{\left[L_{T}, L h, h, b_{0}\right]}$, we may consider a sequence $\left\{u_{T}^{n}\right\} \subset \mathcal{A}_{\left[L_{T}, 2 L h, h, b_{0}\right]} \cap C^{1}(\mathbb{R}, \mathbb{R})$ such that $\lim _{n \rightarrow \infty}\left\|u_{T}^{n}-u_{T}\right\|_{L^{1}}=0$. Let $w^{n}(t, \cdot) \doteq S_{t}\left(w_{0}^{n}\right)$ and $w(t, \cdot) \doteq S_{t}\left(w_{0}\right)$ be the entropy weak solutions of (1) with initial data, respectively, $w_{0}^{n}(\cdot) \doteq u_{T}^{n}(-\cdot)$ and $w_{0}(\cdot) \doteq u_{T}(-\cdot)$. By the $L^{1}$-stability property of the semigroup $S_{t}$ we deduce that $w^{n}(t, \cdot) \rightarrow w(t, \cdot)$ in $L^{1}$ for all $t \geq 0$. On the other hand, by the arguments at point $\mathbf{1}$., we know that the functions defined by setting $u^{n}(t, x) \doteq w^{n}(T-t,-x)$ are entropy weak solutions of (1), which attain the value $u_{T}^{n}$ at time $T$, and whose initial conditions satisfy $u^{n}(0, \cdot) \in \mathcal{C}_{[L, m, M]}$, for all $n$. Thus, it follows that the $L_{l o c}^{1}$-limit $u$ of $u^{n}$ provides an entropy weak solutions of $(1)$ as well, that $u(0, \cdot) \in \mathcal{C}_{[L, m, M]}$, and that $u(T, \cdot)=u_{T}$, completing the proof of the proposition.

\subsection{Proof of Proposition 2}

1. Following a similar strategy as the one pursued in [1], we will establish a lower bound on the covering number $N_{\varepsilon}\left(\mathcal{A}_{[L, m, M, b]}\right)$, by first introducing a two-parameters class of piecewise affine functions in $\mathcal{A}_{[L, m, M, b]}$, and next providing an estimate of the maximum number of such functions contained in a subset of $\mathcal{A}_{[L, m, M, b]}$ having diameter no larger than $2 \varepsilon$. Namely, given any integer $n \geq 2$, and a constant $h>0$, for every $n$-tuple $\iota=\left(\iota_{i}\right)_{i=0, \ldots, n-1} \in\{-1,1\}^{n}$ consider the function $\mathcal{F}_{\iota}: \mathbb{R} \rightarrow[-h, h]$, with support contained in $[-L, L]$, defined by (see Figure 1$)$ :

$$
\mathcal{F}_{\iota}(x)=\left\{\begin{array}{l}
\frac{h n}{2 L}\left(x+L-k \frac{2 L}{n}\right) \quad \text { if } \iota_{k}=1, \\
\frac{h n}{2 L}\left(x+L-(k+1) \frac{2 L}{n}\right) \quad \text { if } \iota_{k}=-1,
\end{array} \quad \forall x \in\left[-L+k \frac{2 L}{n},-L+(k+1) \frac{2 L}{n}\right],\right.
$$

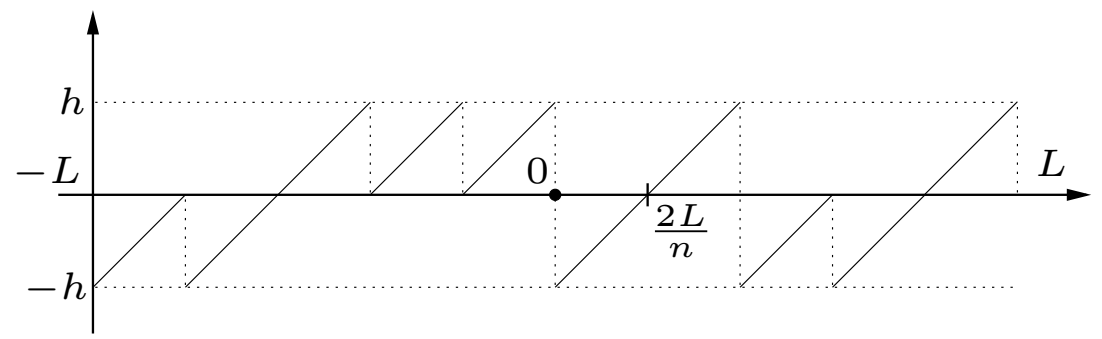

Figure 1: The function $\mathcal{F}_{\iota}$ for $n=10$ and $\iota=(-1,-1,1,1,1,-1,1,-1,-1,1)$

Notice that every $\mathcal{F}_{\iota}, \iota \in\{-1,1\}^{n}$, belongs to $\mathcal{A}_{[L, m, M, b]}$ provided that

$$
h \leq M, \quad h \leq \frac{m}{L}, \quad n h \leq 2 L b .
$$

Moreover, given any $\iota, \bar{\iota} \in\{-1,1\}^{n}$, one has

$$
\left\|\mathcal{F}_{\iota}-\mathcal{F}_{\bar{\iota}}\right\|_{L^{1}}=\frac{2 h L}{n} d(\iota, \bar{\iota}),
$$

where

It follows that

$$
d(\iota, \bar{\iota}) \doteq \operatorname{Card}\left\{k \in\{1, \ldots, n\} \mid \iota_{k} \neq \bar{\iota}_{k}\right\}
$$

$$
\left\|\mathcal{F}_{\iota}-\mathcal{F}_{\bar{\iota}}\right\|_{L^{1}} \leq \varepsilon \quad \Longleftrightarrow \quad d(\iota, \bar{\iota}) \leq \frac{n \varepsilon}{2 h L} .
$$

Notice that, given any fixed $\bar{\iota} \in\{-1,1\}^{n}$, the set $\mathcal{I}_{\bar{\iota}}(\varepsilon)$ of $n$-tuples $\iota \in\{-1,1\}^{n}$ such that $d(\iota, \bar{\iota}) \leq \frac{n \varepsilon}{2 h L}$ depends on $\bar{\iota}$, but the number of elements of $\mathcal{I}_{\bar{\iota}}(\varepsilon)$ is independent of the choice of $\bar{\iota}$. Denote $\mathcal{C}(\varepsilon)$ such a number. By standard combinatorial properties, counting the $n$-tuples that differ for a given number of entries, we compute

$$
\mathcal{C}(\varepsilon)=\sum_{\ell=0}^{\left\lfloor\frac{n \varepsilon}{2 h L}\right\rfloor}\left(\begin{array}{l}
n \\
\ell
\end{array}\right),
$$


where $\lfloor\alpha\rfloor \doteq \max \{z \in \mathbb{Z} \mid z \leq \alpha\}$ denotes the integer part of $\alpha$. In order to provide an estimate of $\mathcal{C}(\varepsilon)$, we rewrite the right-hand side of (39) using the fact that, if $X_{1}, \ldots, X_{n}$ are independent random variables with Bernoulli distribution $\mathbb{P}\left(X_{i}=0\right)=\mathbb{P}\left(X_{i}=1\right)=\frac{1}{2}$, then for any $k \leq n$ one has

$$
\mathbb{P}\left(X_{1}+\cdots+X_{n} \leq k\right)=\frac{1}{2^{n}} \sum_{\ell=0}^{k}\left(\begin{array}{l}
n \\
\ell
\end{array}\right) .
$$

We may estimate the left-hand side of (40) setting $S_{n}=X_{1}+\cdots+X_{n}$, and using Hoeffding's inequality ([8, Theorem 2]) that, for any fixed $\mu>0$, gives

$$
\mathbb{P}\left(S_{n}-\mathbb{E}\left(S_{n}\right) \leq-\mu\right) \leq \exp \left(-\frac{2 \mu^{2}}{n}\right),
$$

where $\mathbb{E}\left(S_{n}\right)$ denotes the expectation of $S_{n}$. Since, by the above assumptions on $X_{1}, \ldots, X_{n}$, we have $\mathbb{E}\left(S_{n}\right)=\frac{n}{2}$, taking $\mu=\frac{n}{2}-\left\lfloor\frac{n \varepsilon}{2 h L}\right\rfloor, k=\left\lfloor\frac{n \varepsilon}{2 h L}\right\rfloor$, and assuming

$$
\frac{n \varepsilon}{2 h L} \leq \frac{n}{2},
$$

we deduce from (39)-(41) that

$$
\begin{aligned}
\frac{1}{2^{n}} \mathcal{C}(\varepsilon) & \leq \exp \left(-2 \frac{\left(\frac{n}{2}-\left\lfloor\frac{n \varepsilon}{2 h L}\right\rfloor\right)^{2}}{n}\right) \\
& \leq \exp \left(-\frac{n}{2}\left(1-\frac{\varepsilon}{h L}\right)^{2}\right) .
\end{aligned}
$$

2. To obtain a large lower bound on the covering number of $\mathcal{A}_{[L, M, m, b]}$, let us maximize the map

$$
\Psi(h, n) \doteq \frac{n}{2}\left(1-\frac{\varepsilon}{h L}\right)^{2},
$$

with the parameters $h, n$, subject to (38) and (42). If we first optimize $\Psi(h, n)$ with respect to $h$ (letting $n$ be sufficiently large so that the first two constraints in (38) be satisfied) we find that the maximum is attained for

$$
h_{n} \doteq \frac{2 b L}{n} .
$$

Next, optimizing $\Psi\left(h_{n}, n\right)$ for $n$ satisfying $(42)$, we deduce that the maximum is attained for

$$
\bar{n} \doteq \frac{2 b L^{2}}{3 \varepsilon} .
$$

One can check that

$$
\frac{\bar{n} \varepsilon}{2 h_{\bar{n}} L}=\frac{\bar{n}}{6}<\frac{\bar{n}}{2}
$$

so that, with $h_{\bar{n}}, \bar{n}$ defined by (44), (45), conditions (38), (42) are both verified provided that $\varepsilon$ satisfies (23). Hence, we deduce from (43) that

$$
\frac{\mathcal{C}(\varepsilon)}{2^{n}} \leq \exp \left(-\Psi\left(h_{\bar{n}}, \bar{n}\right)\right)=\exp \left(-\frac{1}{\varepsilon} \cdot \frac{4 b L^{2}}{27}\right) .
$$

Now observe that any $\varepsilon$-cover of $\mathcal{A}_{[L, M, m, b]}$, in particular, contains the set

$$
\mathcal{F} \doteq\left\{\mathcal{F}_{\iota}: \mathbb{R} \rightarrow\left[-h_{\bar{n}}, h_{\bar{n}}\right] ; \quad \iota \in\{-1,1\}^{n}\right\},
$$

and that each element of this cover contains at most $\mathcal{C}(2 \varepsilon)$ functions of $\mathcal{F}$. Since the cardinality of $\mathcal{F}$ is $2^{n}$, it follows that the number of sets in an $\varepsilon$-cover of $\mathcal{A}_{[L, M, m, b]}$ is at least

$$
N_{\varepsilon}\left(\mathcal{A}_{[L, M, m, b]}\right) \geq \frac{2^{n}}{\mathcal{C}(2 \varepsilon)} \geq \exp \left(\frac{1}{\varepsilon} \cdot \frac{2 b L^{2}}{27}\right)
$$

which yields (24), thus completing the proof of Proposition 2. 


\section{Lower compactness estimates for balance laws}

\subsection{Proof of Theorem 3}

In order to establish Theorem 3, we will make use of a local Oleinik type estimate for balance laws (9). An inequality of this kind was established in [13, Theorem 1.2]. Here, we provide a slightly more accurate estimate, determining how the constant $C$ appearing in [13, Theorem 1.2] depends on the time $t$ and on the set of points $x, y$ for which the inequality holds. For source terms of the form $g=g(u)$, a global Oleinik type estimate was obtained in [7, Section 4]. Recall the notation $k_{a, b, t}(M)$ from $(16)$.

Lemma 1. Let $f: \mathbb{R} \rightarrow \mathbb{R}$ be a twice continuously differentiable map that satisfies (2), and $g: \mathbb{R}^{+} \times$ $\mathbb{R} \times \mathbb{R} \rightarrow \mathbb{R}$ be a continuously differentiable map that satisfies (10), (12). Given $u_{0} \in L^{1}(\mathbb{R}) \cap L^{\infty}(\mathbb{R})$, let $u: \mathbb{R}^{+} \times \mathbb{R} \rightarrow \mathbb{R}$ be the corresponding entropy admissible solution of (9) with initial condition $u(0, \cdot)=u_{0}$, and set

$$
M_{t} \doteq\left\|u_{0}\right\|_{L^{\infty}} \cdot \exp \left(\|\omega\|_{L^{1}(0, t)}\right) \quad t \geq 0
$$

Then, for all $t>0$, and for any given $a, b \in \mathbb{R}, a<b$, there holds

$$
\frac{u(t, y)-u(t, x)}{y-x} \leq \frac{\left(1+c t^{2} k_{a, b, t}\left(M_{t}\right)\right) \cdot \exp \left(\|\omega\|_{L^{1}(0, t)}\right)}{c t} \quad \forall x, y \in[a, b], \quad x<y .
$$

Remark 4. From the proof of the lemma it will be clear that, when the source term has the form $g=g(t, u)$, and satisfies (12), but not necessarily (10), one obtains the global Oleinik estimate

$$
\frac{u(t, y)-u(t, x)}{y-x} \leq \frac{\exp \left(\|\omega\|_{L^{1}(0, t)}\right)}{c t} \quad \forall x, y \in \mathbb{R}, \quad x<y,
$$

which is a bit more accurate than the one provided in [7] (showing that the constant $C$ appearing in [7, Section 4$]$ is precisely $1 /$ c).

Relying on Lemma 1, we will establish the analogous result of Proposition 1 in the case of balance laws, which together with Proposition 2 yields the conclusion of Theorem 3.

Proposition 3. Under the assumptions of Lemma 1, assume that $f, g$ satisfy also (3) and (11), respectively. Then, given any $L, m, M, T>0$, for

$$
h \leq \min \left(M, \frac{m}{2 L}, \frac{L}{8 T\left|f^{\prime \prime}(0)\right|}, \frac{\exp \left(-\|\omega\|_{L^{1}(0, T)}\right)}{8\left|f^{\prime \prime}(0)\right| C T^{2}}\right) \exp \left(-\|\omega\|_{L^{1}(0, T)}\right),
$$

sufficiently small ( $C$ being the constant appearing in (11)), one has

$$
\mathcal{A}_{\left[\widetilde{L}_{T}, L h, h,\left(2 T\left|f^{\prime \prime}(0)\right| \exp \left(\|\omega\|_{L^{1}(0, T)}\right)\right)^{-1}\right]} \subset E_{T}\left(\mathcal{C}_{[L, m, M]}\right)
$$

with

$$
\widetilde{L}_{T} \doteq L-2 T\left|f^{\prime \prime}(0)\right| \exp \left(\|\omega\|_{L^{1}(0, T)}\right) \cdot h
$$

Note that, thanks to (49), the constant $\widetilde{L}_{T}$ given in (51) satisfies $\widetilde{L}_{T} \geq(3 / 4) L$. Hence, relying on (50), and applying (24), with $L=\widetilde{L}_{T}, b=\left(2 T\left|f^{\prime \prime}(0)\right| \exp \left(\|\omega\|_{L^{1}(0, T)}\right)\right)^{-1}$, we recover the estimate (14), which proves Theorem 3.

\subsection{Proof of Lemma 1}

1. It will be sufficient to prove (47) when the initial data $u_{0} \in L^{1}(\mathbb{R}) \cap B V(\mathbb{R})$, since one can then recover $(47)$ for general data $u_{0} \in L^{1}(\mathbb{R}) \cap L^{\infty}(\mathbb{R})$ exploiting the $L^{1}$-continuity of the evolution operator $E_{t}, t>0$, and the lower semicontinuity of the positive variation. Therefore, we may assume that $u(t, \cdot) \doteq$ $E_{t} u_{0} \in B V(\mathbb{R})$ for all $t \geq 0$, and thus we can rely on Dafermos' theory of generalized characteristics (we 
refer to $[4$, Section 11.9]). Moreover, it is not restrictive to suppose that $u(t, \cdot)$ is right continuous, and to establish (47) only at points $x, y \in[a, b]$ where $u(t, \cdot)$ is continuous (since one then derives (47) at the points of discontinuity taking the right limits of $u(t, \cdot))$.

Observe that, if $\xi(\cdot)$ denotes the maximal backward generalized characteristic emanating from a point $(t, x)$, then by [4, Theorem 11.9.1] there is some $C^{1}$ function $v(\cdot)$ that, together with $\xi(\cdot)$, satisfies on $] 0, t[$ the characteristic equation

$$
\left\{\begin{array}{l}
\dot{\xi}(s)=f^{\prime}(v(s)), \\
\dot{v}(s)=g(s, \xi(s), v(s)),
\end{array}\right.
$$

with

$$
\xi(t)=x, \quad v(t)=u(t, x) .
$$

Furthermore, there holds $u(s, \xi(s) \pm)=v(s)$ for all $s \in] 0, t[$, and

$$
u_{0}(\xi(0)-) \leq v(0) \leq u_{0}(\xi(0)+) .
$$

Therefore, since (13), (52) imply

$$
\frac{d}{d s}|v(s)| \leq \omega(s) \cdot|v(s)|,
$$

applying Gronwall's lemma, and using (53), (54), we deduce

$$
|u(t, x)| \leq \max \left\{\left|u_{0}(\xi(0)-)\right|,\left|u_{0}(\xi(0)+)\right|\right\} \cdot \exp \left(\|\omega\|_{L^{1}(0, t)}\right) .
$$

In turn, this yields

$$
\|u(s, \cdot)\|_{L^{\infty}} \leq M_{t} \quad \forall s \in[0, t], \quad t \geq 0,
$$

with $M_{t}$ defined by (46). Relying on (56), and because of (52), we deduce that the set $\Delta_{a, b, t}\left(M_{t}\right)$ defined in (15) is a backward domain of determinacy relative to the interval $[a, b]$ and to the time $t$, since it contains all backward generalized characteristics emanating from points $(t, x), x \in[a, b]$.

2. Fix $t>0, a, b \in \mathbb{R}, a<b$, and consider $x<y$ two points of continuity of $u(t, \cdot)$ inside $[a, b]$. Let $\xi^{x}(\cdot)$ and $\xi^{y}(\cdot)$ be the (unique) backward generalized characteristics (cfr. [4, Theorems 11.9.5]) emanating from $(t, x)$ and $(t, y)$, respectively. By [4, Theorems 11.9.1 \& 11.9.3] there will be some $C^{1}$ functions $v^{x}(\cdot), v^{y}(\cdot)$, so that $\left(\xi^{x}(\cdot), v^{x}(\cdot)\right)$ and $\left(\xi^{y}(\cdot), v^{y}(\cdot)\right)$ satisfy on $] 0, t[$ the characteristic equations (52) with

$$
\xi^{x}(t)=x, \quad v^{x}(t)=u(t, x) \quad \text { and } \quad \xi^{y}(t)=y, \quad v^{y}(t)=u(t, y) .
$$

Observe that, if $u(t, x) \geq u(t, y)$, the inequality (47) is certainly satisfied since its right-hand side is always positive. Therefore, by virtue of (57) we will consider only the case $v^{x}(t)<v^{y}(t)$. Then, set

$$
\left.\bar{\sigma} \doteq \inf \{\sigma \in] 0, t] \mid v^{x}(s)<v^{y}(s) \quad \forall s \in[\sigma, t]\right\},
$$

and observe that, by the strict convexity assumption (2) on $f$, and because of (52), one has

$$
y-x \geq \xi^{y}(s)-\xi^{x}(s) \quad \forall s \in[\bar{\sigma}, t] .
$$

Moreover, since $\xi^{x}(\cdot)$ and $\xi^{y}(\cdot)$ do not cross at any time $\left.\left.s \in\right] 0, t\right]$ (cfr. [4, Section 11.9]), it follows that

$$
\xi^{y}(s) \geq \xi^{x}(s) \quad \forall s \in[0, t] .
$$

Then, relying on (12), (52), (56), (59), and recalling (16), we deduce that for all $s \in] \bar{\sigma}, t[$, there holds

$$
\begin{aligned}
\dot{v}^{y}(s)-\dot{v}^{x}(s) & =g\left(s, \xi^{y}(s), v^{y}(s)\right)-g\left(s, \xi^{y}(s), v^{x}(s)\right)+g\left(s, \xi^{y}(s), v^{x}(s)\right)-g\left(s, \xi^{x}(s), v^{x}(s)\right) \\
& \leq \omega(s)\left(v^{y}(s)-v^{x}(s)\right)+k_{a, b, t}\left(M_{t}\right)\left(\xi^{y}(s)-\xi^{x}(s)\right) \\
& \leq \omega(s)\left(v^{y}(s)-v^{x}(s)\right)+k_{a, b, t}\left(M_{t}\right)(y-x) .
\end{aligned}
$$


Hence, using Gronwall's lemma, from (61) we derive

$$
\begin{aligned}
v^{y}(s)-v^{x}(s) & \geq\left(v^{y}(t)-v^{x}(t)\right) \exp \left(-\int_{s}^{t} \omega(\tau) d \tau\right)-(t-s) k_{a, b, t}\left(M_{t}\right)(y-x) \\
& \geq\left(v^{y}(t)-v^{x}(t)\right) \exp \left(-\int_{0}^{t} \omega(\tau) d \tau\right)-t k_{a, b, t}\left(M_{t}\right)(y-x)
\end{aligned} \quad \forall s \in[\bar{\sigma}, t] .
$$

Two cases now may occur. If $\bar{\sigma}>0$, by the definition (58) and because of the continuity of $v^{x}(\cdot), v^{y}(\cdot)$ it follows that $v^{x}(\bar{\sigma})=v^{y}(\bar{\sigma})$, which together with (62), and recalling (57), yields

$$
u(t, y)-u(t, x) \leq t k_{a, b, t}\left(M_{t}\right) \exp \left(\int_{0}^{t} \omega(\tau) d \tau\right)(y-x) .
$$

Instead, if $\bar{\sigma}=0$, using (62), and relying on (2), (60), we deduce

$$
\begin{aligned}
0 & \leq \xi^{y}(0)-\xi^{x}(0) \\
& =y-x-\int_{0}^{t}\left(f^{\prime}\left(v^{y}(s)\right)-f^{\prime}\left(v^{x}(s)\right)\right) d s \\
& \leq y-x-c \int_{0}^{t}\left(v^{y}(s)-v^{x}(s)\right) d s \\
& \leq\left(1+c t^{2} k_{a, b, t}\left(M_{t}\right)\right)(y-x)-c t\left(v^{y}(t)-v^{x}(t)\right) \exp \left(-\int_{0}^{t} \omega(\tau) d \tau\right),
\end{aligned}
$$

which, by virtue of (57), yields

$$
u(t, y)-u(t, x) \leq \frac{\left(1+c t^{2} k_{a, b, t}\left(M_{t}\right)\right)(y-x)}{c t} \exp \left(\int_{0}^{t} \omega(\tau) d \tau\right) .
$$

Hence, from (63), (64) we recover the inequality (47) concluding the proof of the lemma.

Notice that the assumption (10) was used, in conjunction with (12), only to establish the a-priori bound (56) on the $L^{\infty}$ norm of the solution, which in turn was needed to define a bound on $D_{x} g$ over a domain of determinacy of the solution. Therefore, as observed in Remark 4 , the conclusion of the lemma continues to hold (with $k_{a, b, t}\left(M_{t}\right)=0$ in (47)) in the case the source term $g=g(t, u)$ satisfies only the assumption (12).

\subsection{Proof of Proposition 3}

In the same spirit of the proof of Proposition 1, we will first show that

$$
\mathcal{A}_{\left[\widetilde{L}_{T}, 2 L h, h,\left(2 T\left|f^{\prime \prime}(0)\right|\right)^{-1}\right]} \cap C^{1}(\mathbb{R}) \subset E_{T}\left(\mathcal{C}_{[L, m, M]}\right) .
$$

To this end, given

$$
u_{T} \in \mathcal{A}_{\left[\widetilde{L}_{T}, 2 L h, h,\left(2 T\left|f^{\prime \prime}(0)\right| \exp \left(\|\omega\|_{L^{1}(0, T)}\right)\right)^{-1}\right]} \cap C^{1}(\mathbb{R}),
$$

setting

$$
\widetilde{g}(t, x, u) \doteq-g(T-t,-x, u),
$$

we consider the entropy weak solution $w(t, x) \doteq \widetilde{E}_{t} w_{0}$ of

$$
w_{t}+f(w)_{x}=\widetilde{g}(t, x, w),
$$

with initial data

$$
w_{0}(x) \doteq u_{T}(-x) \quad \forall x \in \mathbb{R} .
$$

By the estimate (56) established in the proof of Lemma 1, and because of (49), (66), (67), there holds

$$
\begin{aligned}
\|w(t, \cdot)\|_{L^{\infty}} & \leq \exp \left(\|\omega\|_{L^{1}(0, T)}\right)\left\|u_{T}\right\|_{L^{\infty}} \\
& \leq \exp \left(\|\omega\|_{L^{1}(0, T)}\right) h \leq M
\end{aligned}
$$


Next, observe that the function

$$
u(t, x) \doteq w(T-t,-x), \quad \forall(t, x) \in[0, T] \times \mathbb{R},
$$

is a weak distributional solution of (9), which, by (67), satisfies

$$
u(T, \cdot)=u_{T} .
$$

Moreover, if we show that for all $t \in[0, T[, x \mapsto w(t, x)$ is smooth on $\mathbb{R}$, it would follow that $u(t, x)$ provides an entropy admissible weak solution of $(9)$ on $[0, T] \times \mathbb{R}$. To establish the smoothness of $w(t, \cdot)$, recalling that by $(66),(67)$ one has $w_{0}(\cdot) \in C^{1}(\mathbb{R})$, we will show as in the proof of Proposition 1 that, letting $T_{1}$ be the quantity defined by $(30)$, there holds $T_{1}=T$. To this purpose, notice first that the Oleinik type estimate in Lemma 1 provides an upper bound for $w_{x}(t, \cdot), t>0$, on any bounded set of $\mathbb{R}$. Therefore, because of (31), in order to prove that $T_{1}=T$ it will be sufficient to establish a lower bound for $w_{x}$. With this aim, observing that the function $v(t, x) \doteq w_{x}(t, x)$, is the unique broad solution on $\left[0, T_{1}[\times \mathbb{R}\right.$ of the semilinear equation

$$
v_{t}(t, x)+f^{\prime}(w(t, x)) \cdot v_{x}(t, x)=\widetilde{g}_{x}(t, x, w(t, x))+\widetilde{g}_{u}(t, x, w(t, x)) \cdot v(t, x)-f^{\prime \prime}(w(t, x)) \cdot v(t, x)^{2}
$$

(see [2, Theorem 3.6]), we deduce that the value $z(t) \doteq v(t, x(t))$ of $v$ along a characteristic $x(\cdot)$ of $(9)$ satisfies the equation

$$
\dot{z}(t)=\widetilde{g}_{x}(t, x(t), w(t, x(t)))+\widetilde{g}_{u}(t, x(t), w(t, x(t))) \cdot z(t)-f^{\prime \prime}(w(t, x(t))) \cdot z^{2}(t), \quad t \in\left[0, T_{1}[.\right.
$$

Notice that, by the continuity of $f^{\prime \prime}$ we may assume that

$$
f^{\prime \prime}(\rho) \leq \frac{3}{2} f^{\prime \prime}(0) \quad \text { for } \quad|\rho| \leq \rho_{h} \doteq \exp \left(\|\omega\|_{L^{1}(0, T)}\right) \cdot h,
$$

when $h>0$ is sufficiently small. Moreover, observe that, by the continuity of $z(\cdot)$, and because of (66), (67), it will be sufficient to provide a uniform lower bound for $z(\cdot)$ on every compact interval $\left[\tau_{1}, \tau_{2}\right] \subset$ $\left[0, T_{1}[\right.$, during which $z(t)<0$, and such that

$$
z\left(\tau_{1}\right) \geq-\left(2 T f^{\prime \prime}(0) \exp \left(\|\omega\|_{L^{1}(0, T)}\right)\right)^{-1} .
$$

Hence, fix any such interval $\left[\tau_{1}, \tau_{2}\right]$, and relying on (10), (11), (12), (68), (70), derive from (69) the lower bound

$$
\dot{z}(t) \geq-C \cdot \rho_{h}+\omega(t) \cdot z(t)-\frac{3}{2} f^{\prime \prime}(0) \cdot z^{2}(t), \quad t \in\left[\tau_{1}, \tau_{2}\right] .
$$

Next, setting

$$
z_{1}(t) \doteq \exp \left(-\int_{0}^{t} \omega(s) d s\right) \cdot z(t)
$$

we deduce from (72) that there holds

$$
\dot{z}_{1}(t) \geq-\frac{3}{2} f^{\prime \prime}(0) \exp \left(\|\omega\|_{L^{1}(0, T)}\right) \cdot\left(\frac{2 C h}{3 f^{\prime \prime}(0)}+z_{1}^{2}(t)\right), \quad t \in\left[\tau_{1}, \tau_{2}\right],
$$

which yields

$\arctan \left(\sqrt{\frac{3 f^{\prime \prime}(0)}{2 C h}} \cdot z_{1}(t)\right) \geq \arctan \left(\sqrt{\frac{3 f^{\prime \prime}(0)}{2 C h}} \cdot z_{1}\left(\tau_{1}\right)\right)-\sqrt{\frac{3 f^{\prime \prime}(0) C h}{2}} \exp \left(\|\omega\|_{L^{1}(0, T)}\right) \cdot T, \quad t \in\left[\tau_{1}, \tau_{2}\right]$.

Then, recalling (71), and setting $\xi_{h, T} \doteq \exp \left(\|\omega\|_{L^{1}(0, T)}\right) \sqrt{f^{\prime \prime}(0) C h} \cdot T$, we obtain

$$
\arctan \left(\sqrt{\frac{3 f^{\prime \prime}(0)}{2 C h}} \cdot z_{1}(t)\right) \geq-\arctan \left(\sqrt{\frac{3}{8}} \cdot \frac{1}{\xi_{h, T}}\right)-\sqrt{\frac{3}{2}} \cdot \xi_{h, T}, \quad t \in\left[\tau_{1}, \tau_{2}\right],
$$

which, in turn, implies

$$
\arctan \left(\sqrt{\frac{3 f^{\prime \prime}(0)}{2 C h}} \cdot z_{1}(t)\right) \geq-\frac{\pi}{2}+\arctan \left(\sqrt{\frac{8}{3}} \cdot \xi_{h, T}\right)-\sqrt{\frac{3}{2}} \cdot \xi_{h, T}, \quad t \in\left[\tau_{1}, \tau_{2}\right] .
$$


On the other hand, one can easily check that

$$
\arctan \left(\sqrt{\frac{8}{3}} \xi\right)-\sqrt{\frac{3}{2}} \xi \geq \arctan \left(\sqrt{\frac{1}{3}}\right)-\frac{\sqrt{3}}{4}=\frac{2 \pi-3 \sqrt{3}}{12}>0 \quad \forall \xi \in[0,1 / \sqrt{8}] .
$$

Therefore, since (49) implies that $\xi_{h, T} \leq 1 / \sqrt{8}$, we deduce from (75)-(76) that there will be some constant $C_{h, T}>0$ such that, for every interval $\left[\tau_{1}, \tau_{2}\right] \subset\left[0, T_{1}[\right.$, where $z(t)<0$, and for which there holds $(71)$, one has $z(t)>-C_{h, T}$, for all $t \in\left[\tau_{1}, \tau_{2}\right]$. This yields a uniform lower bound for $w_{x}$ on $\left[0, T_{1}[\times \mathbb{R}\right.$, which by the above observations implies that $T_{1}=T$, and that $u(t, \cdot)=w(T-t,-\cdot) \in C^{1}(\mathbb{R})$, for all $\left.t \in\right] 0, T[$.

To complete the proof of $u_{T} \in E_{T}\left(\mathcal{C}_{[L, m, M]}\right)$, we only need to show that the initial condition $u(0, \cdot)=$ $w(T,-\cdot)$ is an element of the set $\mathcal{C}_{[L, m, M]}$ defined in (5). By the estimates (68) we already know that $u(0, \cdot)$ satisfies the $L^{\infty}$ bound of $\mathcal{C}_{[L, m, M]}$. On the other hand, observing that the solution $w$ propagates along classical characteristics, and relying on (3), (66), (67), (68), (70), we derive (for $h$ sufficiently small) the bound on the support of $w$ :

$$
\operatorname{Supp}(w(t, \cdot)) \subset[-l(t), l(t)], \quad \forall t \in[0, T],
$$

with

$$
\begin{aligned}
l(t) & \doteq \widetilde{L}_{T}+t \rho_{h}\left\|f^{\prime \prime}\right\|_{L^{\infty}\left(-\rho_{h}, \rho_{h}\right)} \\
& \leq \widetilde{L}_{T}+\frac{3}{2} T\left|f^{\prime \prime}(0)\right| \exp \left(\|\omega\|_{L^{1}(0, T)}\right) \cdot h,
\end{aligned}
$$

which, in turn, recalling (51), yields

$$
\operatorname{Supp}(u(0, \cdot))=\operatorname{Supp}(w(T,-\cdot)) \in[-L, L] .
$$

Finally, relying on (49), (68), (78), we derive

$$
\|w(t, \cdot)\|_{L^{1}} \leq 2 L \exp \left(\|\omega\|_{L^{1}(0, T)}\right) h \leq m \quad \forall t \in[0, T] .
$$

Hence, (68), (78), (79) together yield $u(0, \cdot) \in \mathcal{C}_{[L, m, M]}$, which concludes the proof of (65).

The proof of Proposition 3 is then completed with the same density argument performed at point 2. of the proof of Proposition 1, relying on the $L^{1}$ stability property of the evolution operator $E_{t}$.

\section{Upper compactness estimates for balance laws}

\subsection{Proof of Theorem 4}

Following the arguments of De Lellis and Golse in [5], we shall establish an upper estimate on the $\varepsilon$ entropy of $E_{T}\left(\mathcal{C}_{[L, m, M]}\right)$ relying on the upper bound on the $\varepsilon$-entropy of a class of nondecreasing functions provided by:

Lemma 2. ([5, Lemma 3.1]) Given any, $L, V>0$, setting

$$
\mathcal{I}_{[L, V]} \doteq\{v:[0, L] \rightarrow[0, V] \mid v \text { is nondecreasing }\},
$$

for $0<\varepsilon<\frac{L V}{6}$, there holds

$$
H_{\varepsilon}\left(\mathcal{I}_{[L, V]} \mid L^{1}([0, L])\right) \leq 4\left\lfloor\frac{L V}{\varepsilon}\right\rfloor
$$

In order to derive an a-priori bound on the support of solutions to balance laws in terms of the $L^{1}$ norm of their initial data, we will use the next technical lemma whose proof is provided below.

Lemma 3. Given $v \in \mathrm{BV}(\mathbb{R})$, compactly supported and satisfying

$$
D v \leq B \quad \text { in the sense of measures, }
$$

for some constant $B>0$, there holds

$$
\|v\|_{L^{\infty}} \leq \sqrt{2 B\|v\|_{L^{1}}} .
$$


Now, supposing Lemma 3 proven, one establishes Theorem 4 as follows.

1. Given any $u_{0} \in \mathcal{C}_{[L, m, M]}$, we shall first derive an a-priori bound on the $L^{1}$ norm of the corresponding entropy weak solution $u(t, \cdot) \doteq E_{t} u_{0}$ of $(9)$, relying on the Oleinik type estimates provided by Lemma 1 . To this end, observe that by standard arguments for conservation laws (e.g. cfr. proof of [4, Theorem 11.8.2]), and thanks to (13), we find

$$
\frac{d}{d t}\|u(t, \cdot)\|_{L^{1}} \leq\|g(t, \cdot, u(t, \cdot))\|_{L^{1}} \leq \omega(t) \cdot\|u(t, \cdot)\|_{L^{1}} .
$$

Hence, applying Gronwall's lemma, and by the definition (5), we deduce

$$
\|u(t, \cdot)\|_{L^{1}} \leq \exp \left(\|\omega\|_{L^{1}(0, t)}\right) \cdot\left\|u_{0}\right\|_{L^{1}} \leq \exp \left(\|\omega\|_{L^{1}(0, T)}\right) \cdot m \quad \forall t \in[0, T] .
$$

Moreover, as discussed in the proof of Proposition 3, because of (5) and (56), we have

$$
\|u(t, \cdot)\|_{L^{\infty}} \leq M_{T} \doteq \exp \left(\|\omega\|_{L^{1}(0, T)}\right) \cdot M \quad \forall t \in[0, T],
$$

and with (3), (54) and (55), that

$$
\operatorname{Supp}(u(t, \cdot)) \subset[-l(t), l(t)] \quad \forall t \in[0, T],
$$

with

$$
l(t) \leq L+\left\|f^{\prime \prime}\right\|_{L^{\infty}\left(-M_{T}, M_{T}\right)} \int_{0}^{T}\|u(t, \cdot)\|_{L^{\infty}} d t .
$$

On the other hand, observe that (83)-(85) and the estimate (47) imply $u(t, \cdot) \in \mathrm{BV}(\mathbb{R})$, and

$$
\left.\left.D u(t) \leq \frac{\left(1+c t^{2} K_{L, T}\right) \cdot \exp \left(\|\omega\|_{L^{1}(0, T)}\right)}{c t} \quad \forall t \in\right] 0, T\right],
$$

with

$$
K_{L, T} \doteq k_{-L_{T}, L_{T}, T}\left(M_{T}\right), \quad L_{T} \doteq L+\left\|f^{\prime \prime}\right\|_{L^{\infty}\left(-M_{T}, M_{T}\right)} \cdot M_{T} T,
$$

$\left(k_{-L_{T}, L_{T}, T}\left(M_{T}\right)\right.$ being defined in (16)). Hence, applying Lemma 3 and relying on (82), (86), we derive

$$
\left.\left.\|u(t, \cdot)\|_{L^{\infty}} \leq \sqrt{\frac{2 m}{c}} \cdot \exp \left(\|\omega\|_{L^{1}(0, T)}\right) \sqrt{\frac{\left(1+c t^{2} K_{L, T}\right)}{t}} \quad \forall t \in\right] 0, T\right],
$$

which, together with (85), yields

$$
\begin{aligned}
l(t) & \leq L+\left\|f^{\prime \prime}\right\|_{L^{\infty}\left(-M_{T}, M_{T}\right)} \sqrt{\frac{2 m}{c}} \cdot \exp \left(\|\omega\|_{L^{1}(0, T)}\right) \int_{0}^{T} \sqrt{\frac{\left(1+c t^{2} K_{L, T}\right)}{t}} d t \\
& \leq L(T) \doteq L+2\left\|f^{\prime \prime}\right\|_{L^{\infty}\left(-M_{T}, M_{T}\right)} \sqrt{\frac{2 m T}{c}}\left[1+T \sqrt{c K_{L, T}}\right] \cdot \exp \left(\|\omega\|_{L^{1}(0, T)}\right) .
\end{aligned}
$$

Then, thanks to (2) and (87)-(88), we deduce

$$
\|u(T, \cdot)\|_{L^{\infty}} \leq \frac{L(T)}{2 c T} .
$$

2. In connection with $u_{T} \doteq E_{T} u_{0}$, consider now the function

$$
u_{\sharp}(x) \doteq \frac{\left(1+c T^{2} K_{L, T}\right) \exp \left(\|\omega\|_{L^{1}(0, T)}\right)}{c T} x+\frac{L(T)}{2 c T}-u_{T}(x-L(T)), \quad x \in[0,2 L(T)] .
$$


By virtue of $(86), u_{\sharp}$ is nondecreasing and, thanks to $(89)$, satisfies

$$
0 \leq u^{\sharp}(x) \leq \frac{\left(1+2\left(1+c T^{2} K_{L, T}\right) \exp \left(\|\omega\|_{L^{1}(0, T)}\right)\right) L(T)}{c T} \quad \forall x \in[0,2 L(T)] .
$$

Hence, one has

$$
u^{\sharp} \in \mathcal{I}_{\left[2 L(T),\left(1+2\left(1+c T^{2} K_{L, T}\right) \exp \left(\|\omega\|_{L^{1}(0, T)}\right)\right) \frac{L(T)}{c T}\right]} \cdot
$$

Since $u^{\sharp}$ is obtained from the restriction of $u_{T}$ to $[-L(T), L(T)]$ by a change of sign, a translation by a fixed function, and a shift of a fixed constant, it follows that the $\varepsilon$-entropy relative to $L^{1}(0,2 L(T))$ of the set gathering all functions $u^{\sharp}$ obtained from $E_{T} u_{0}, u_{0} \in \mathcal{C}_{[L, m, M]}$, in this way, equals the one of $E_{T}\left(\mathcal{C}_{[L, m, M]}\right)$ relative to $L^{1}(-L(T), L(T))$. Therefore, the conclusion of Theorem 4 follows by an application of Lemma 2, and observing that, because of (84) and (88), one clearly has

$$
H_{\varepsilon}\left(E_{T}\left(\mathcal{C}_{[L, m, M]}\right) \mid L^{1}(\mathbb{R})\right)=H_{\varepsilon}\left(E_{T}\left(\mathcal{C}_{[L, m, M]}\right) \mid L^{1}(-L(T), L(T))\right) .
$$

\subsection{Proof of Lemma 3}

We shall first establish the conclusion of the Lemma 3 for compactly supported functions $v$, that belong to $C_{0}^{\infty}(\mathbb{R})$, and thus by $(80)$ satisfy $v^{\prime}(x) \leq B$ for all $x \in \mathbb{R}$. Assume that $\operatorname{Supp}(v) \subset[-L, L]$, and consider a point $\bar{x} \in[-L, L]$ such that $|v(\bar{x})|=\|v\|_{\infty}$. We discuss two cases according to the sign of $v(\bar{x})$.

If $v(\bar{x})>0$, defining $y \doteq \min \{x \in[-L, \bar{x}] \mid v>0$ on $] x, \bar{x}]\}$, one has $v(y)=0$, and

$$
v^{2}(\bar{x})=2 \int_{y}^{\bar{x}} v(x) v^{\prime}(x) d x \leq 2 B \int_{y}^{\bar{x}} v(x) d x \leq 2 B\|v\|_{L^{1}(\mathbb{R})} .
$$

If $v(\bar{x})<0$, defining $y \doteq \max \{x \in[\bar{x}, L] \mid v<0$ on $[\bar{x}, x[\}$, one has

$$
v^{2}(\bar{x})=-2 \int_{\bar{x}}^{y} v(x) v^{\prime}(x) d x \leq-2 B \int_{y}^{\bar{x}} v(x) d x \leq 2 B\|v\|_{L^{1}(\mathbb{R})} .
$$

Hence, in both cases we get the estimate (81) when $v$ is smooth.

For general $v \in \mathrm{BV}(\mathbb{R})$ satisfying the assumptions of the lemma, consider $\rho \in C_{0}^{\infty}(\mathbb{R})$, with $\rho \geq 0$ and $\int_{\mathbb{R}} \rho=1$, define the mollifier $\rho_{\nu}(x) \doteq \frac{1}{\nu} \rho\left(\frac{x}{\nu}\right)$, for $\nu>0$, and then introduce a smooth approximation of $v$ setting

$$
v_{\nu} \doteq \rho_{\nu} * v
$$

Observe that, by standard properties of convolutions, and applying the integration-by-parts formula for BV functions, relying on (80) one gets

$$
v_{\nu}^{\prime}-B=\rho_{\nu}^{\prime} * v-\rho_{\nu} * B=\rho_{\nu} *(D v-B) \leq 0 .
$$

Hence, by the above conclusion we can apply $(81)$ to $v_{\nu}$, finding

$$
\left\|v_{\nu}\right\|_{L^{\infty}} \leq \sqrt{2 B\left\|v_{\nu}\right\|_{L^{1}}}
$$

Since $v_{\nu} \rightarrow v$ in $L^{1}(\mathbb{R})$ as $\nu \rightarrow 0^{+}$, and because there holds $\left\|v_{\nu}\right\|_{\infty} \rightarrow\|v\|_{\infty}$ as $\nu \rightarrow 0^{+}$, we then recover from (90) the estimate (81) for $v$, thus completing the proof of the lemma.

\subsection{Proof of Remark 3}

Let us discuss the case when $g=g(t, u)$ satisfies (12), but does not necessarily satisfy (10). As observed in Remark 4, in this case we can still rely on the Oleinik type estimate (48). On the other hand, the solution of (9) will be in general not compactly supported, since the solution with zero initial data will be in general different from zero. Then, to establish the same type of estimates of Theorem 3 and Theorem 4 , 
it will be more appropriate to consider the $\varepsilon$-entropy of the set of functions $E_{T}\left(\mathcal{C}_{[L, m, M]}\right)-E_{T} 0$ (which are compactly supported by the finite speed of propagation of solutions along characteristics) rather than the $\varepsilon$-entropy of $E_{T}\left(\mathcal{C}_{[L, m, M]}\right)$. Clearly, the two sets have the same $\varepsilon$-entropy, being obtained one from the other by a translation.

1. Upper estimate. Observe now that, if $t \mapsto v^{0}(t)$ denotes the solution of the Cauchy problem

$$
\dot{v}=g(t, v), \quad v(0)=0,
$$

then the function defined by setting $v(t, x) \doteq v^{0}(t)$, for all $x \in \mathbb{R}$, results to be the (admissible) solution of (9) with initial data $u_{0} \equiv 0$. Hence, one has

$$
E_{t} 0=v^{0}(t), \quad \forall t>0 .
$$

Thus, setting

$L_{1}(t) \doteq \inf \left\{x \in \operatorname{Supp}\left(E_{t} u_{0}-E_{t} 0\right) \mid u_{0} \in \mathcal{C}_{[L, m, M]}\right\}, \quad L_{2}(t) \doteq \sup \left\{x \in \operatorname{Supp}\left(E_{t} u_{0}-E_{t} 0\right) \mid u_{0} \in \mathcal{C}_{[L, m, M]}\right\}$,

for every $u_{0} \in \mathcal{C}_{[L, m, M]}$ there holds

$$
E_{t} u_{0}(x)=v^{0}(t) \quad \forall t>0, \forall x<L_{1}(t) \text { or } x>L_{2}(t) .
$$

Relying on (12), (94), one then deduces as in the proof of Theorem 4 that

$$
\frac{d}{d t}\left\|E_{t} u_{0}-E_{t} 0\right\|_{L^{1}} \leq\left\|g\left(t, E_{t} u_{0}\right)-g\left(t, E_{t} 0\right)\right\|_{L^{1}} \leq \omega(t) \cdot\left\|E_{t} u_{0}-E_{t} 0\right\|_{L^{1}},
$$

which, in turn, yields the estimate on the $L^{1}$ norm

$$
\left\|E_{t} u_{0}-E_{t} 0\right\|_{L^{1}} \leq \exp \left(\|\omega\|_{L^{1}(0, t)}\right) \cdot\left\|u_{0}\right\|_{L^{1}} \leq \exp \left(\|\omega\|_{L^{1}(0, T)}\right) \cdot m \quad \forall t \in[0, T] .
$$

With similar arguments, one can derive as in the proof of Lemma 1 the a-priori bound on the $L^{\infty}$ norm

$$
\left\|E_{t} u_{0}-E_{t} 0\right\|_{L^{\infty}} \leq \exp \left(\|\omega\|_{L^{1}(0, t)}\right) \cdot\left\|u_{0}\right\|_{L^{\infty}} \leq M_{T} \doteq \exp \left(\|\omega\|_{L^{1}(0, T)}\right) \cdot M \quad \forall t \in[0, T] .
$$

On the other hand, applying Lemma 3, and thanks to (48) and (95), we derive

$$
\left.\left.\left\|E_{t} u_{0}-E_{t} 0\right\|_{L^{\infty}} \leq \sqrt{\frac{2 m}{c t}} \cdot \exp \left(\|\omega\|_{L^{1}(0, T)}\right) \quad \forall t \in\right] 0, T\right] .
$$

Given any $u_{1}, u_{2} \in \mathcal{C}_{[L, m, M]}$, we introduce

$$
l_{1}\left(u_{1}, t\right) \doteq \inf \operatorname{Supp}\left(E_{t} u_{1}-E_{t} 0\right) \text { and } l_{2}\left(u_{2}, t\right) \doteq \sup \operatorname{Supp}\left(E_{t} u_{2}-E_{t} 0\right) .
$$

As in the proof of Lemma 1, we will make use of generalized characteristics (see again [4, Section 11.9]). We consider the maximal backward characteristic emanating from $\left(t, l_{1}\left(u_{1}, t\right)\right)$ associated to $E_{(\cdot)} u_{1}$, denoted $\xi_{1}(\cdot)$, and the minimal backward characteristic emanating from $\left(t, l_{2}\left(u_{2}, t\right)\right)$ associated to $E_{(\cdot)} u_{2}$, denoted $\xi_{2}(\cdot)$. Then there are some $C^{1}$ functions $v_{1}(\cdot)$ and $v_{2}(\cdot)$ so that $\left(\xi_{1}(\cdot), v_{1}(\cdot)\right)$ and $\left(\xi_{2}(\cdot), v_{2}(\cdot)\right)$ satisfy on $] 0, t[$ the characteristic equation $(52)$, with $g(s, v(s))$ in place of $g(s, \xi(s), v(s))$, and with $\xi_{1}(t)=l_{1}\left(u_{1}, t\right), v_{1}(t)=E_{t} u_{1}\left(l_{1}\left(u_{1}, t\right)\right)$, and $\xi_{2}(t)=l_{2}\left(u_{2}, t\right), v_{2}(t)=E_{t} u_{2}\left(l_{2}\left(u_{2}, t\right)\right)$. Observe that by the properties of the characteristics one clearly has $-L \leq \xi_{i}(0) \leq L, i=1,2$, and

$$
\left.\left|v_{i}(s)\right|=\left|E_{s}\left(u_{i}\right)\left(\xi_{i}(s)\right)\right| \leq\left\|E_{s}\left(u_{i}\right)\right\|_{L^{\infty}} \quad \forall s \in\right] 0, t[, \quad i=1,2 .
$$

Moreover, using that $g(\cdot, 0) \in L_{l o c}^{1}$, and applying Gronwall's inequality we find

$$
\left|v^{0}(s)\right| \leq \exp \left(\|\omega\|_{L^{1}(0, t)}\right) \cdot\|g(\cdot, 0)\|_{L^{1}(0, t)} \quad \forall s \in[0, t] .
$$

Hence, relying on (92), (96), (98), we derive the estimates

$$
\max \left(\left|v_{1}(s)\right|,\left|v_{2}(s)\right|\right) \leq M_{T}^{g} \doteq \exp \left(\|\omega\|_{L^{1}(0, T)}\right)\left(M+\|g(\cdot, 0)\|_{L^{1}(0, T)}\right) \quad \forall s \in[0, t]
$$


and using (3),

$$
\left|l_{2}\left(u_{2}, t\right)-l_{1}\left(u_{1}, t\right)\right| \leq 2 L+\left\|f^{\prime \prime}\right\|_{L^{\infty}\left(-M_{T}^{g}, M_{T}^{g}\right)} \int_{0}^{t}\left|v_{2}(s)-v_{1}(s)\right| d s .
$$

On the other hand, thanks to (97), we get

$$
\begin{aligned}
\left|v_{2}(s)-v_{1}(s)\right| & \leq\left\|E_{s}\left(u_{2}\right)-E_{s}(0)\right\|_{L^{\infty}}+\left\|E_{s}\left(u_{1}\right)-E_{s}(0)\right\|_{L^{\infty}} \\
& \left.\left.\leq 2 \sqrt{\frac{2 m}{c s}} \cdot \exp \left(\|\omega\|_{L^{1}(0, T)}\right) \quad \forall s \in\right] 0, t\right] .
\end{aligned}
$$

Recalling (93), by the arbitrariness of $u_{1}, u_{2} \in \mathcal{C}_{[L, m, M]}$, we deduce from (99), (100) the estimate

$$
L_{2}(t)-L_{1}(t) \leq 2 L(T) \doteq 2 L+4\left\|f^{\prime \prime}\right\|_{L^{\infty}\left(-M_{T}^{g}, M_{T}^{g}\right)} \sqrt{\frac{2 m T}{c}} \cdot \exp \left(\|\omega\|_{L^{1}(0, T)}\right) \quad \forall t \in[0, T],
$$

which in turn, together with (2), (97), yields

$$
\left\|E_{T} u_{0}-E_{T} 0\right\|_{L^{\infty}} \leq \frac{L(T)}{2 c T} .
$$

Then, for any given $u_{0} \in \mathcal{C}_{[L, m, M]}$, setting $u_{T} \doteq E_{T} u_{0}-E_{T} 0$, we consider the function

$$
u_{\sharp}(x) \doteq \frac{\exp \left(\|\omega\|_{L^{1}(0, T}\right)}{c T} x+\frac{L(T)}{2 c T}-u_{T}\left(x+L_{1}(T)\right), \quad x \in[0,2 L(T)],
$$

that results to be an element of $\mathcal{I}_{\left[2 L(T), \frac{L(T)}{c T}\left(1+2 \exp \left(\|\omega\|_{L^{1}(0, T)}\right)\right]\right.}$, by virtue of (48) and (101). With the same arguments of the proof of Theorem 4, applying Lemma 2 , we thus obtain the estimate (17).

2. Lower estimate. For what concerns the lower bound on the $\varepsilon$-entropy of the set of solutions of (9), with $g=g(t, u)$ satisfying (12) together with $g(\cdot, 0) \in L_{l o c}^{1}$, following the same lines of the proof of Proposition 3 one can show that, for

$$
h \leq\left(1, M, \frac{m}{L}, \frac{L}{4 T\left\|f^{\prime \prime}\right\|_{L^{\infty}\left(-G_{T}, G_{T}\right)}}\right) \exp \left(-\|\omega\|_{L^{1}(0, T)}\right),
$$

sufficiently small, there holds

$$
\mathcal{A}_{T} \doteq \mathcal{A}_{\left[\widetilde{L}_{T}, L h, h,\left(T\left\|f^{\prime \prime}\right\|_{L^{\infty}\left(-G_{T}, G_{T}\right)} \exp \left(\|\omega\|_{L^{1}(0, T)}\right)\right)^{-1}\right]} \subseteq \mathcal{T}_{\tau_{T}}\left(E_{T}\left(\mathcal{C}_{[L, m, M]}\right)\right)-E_{T} 0,
$$

with

$$
\widetilde{L}_{T} \doteq L-T\left\|f^{\prime \prime}\right\|_{L_{\left(-G_{T}, G_{T}\right)}^{\infty}} \cdot \exp \left(\|\omega\|_{L^{1}(0, T)}\right) h,
$$

where $G_{T}$ is defined by (19), and $\mathcal{T}_{\tau_{T}}$ denotes the shift operator

$$
u(x) \mapsto \mathcal{T}_{\tau_{T}}(u)(x) \doteq u\left(x-\tau_{T}\right),
$$

associated to some constant $\tau_{T}$ to be defined later (see (110)). By the same density argument as in Propositions 1 and 3, it is sufficient to prove, instead of (103), that

$$
\mathcal{A}_{T} \cap C^{1}(\mathbb{R}) \subseteq \mathcal{T}_{\tau_{T}}\left(E_{T}\left(\mathcal{C}_{[L, m, M]}\right)\right)-E_{T} 0 .
$$

Now, given any $u_{T} \in C^{1}(\mathbb{R}) \cap \mathcal{A}_{T}$, consider the entropy weak solution $w(t, x) \doteq \widetilde{E}_{t} w_{0}$ of

$$
w_{t}+f(w)_{x}=\widetilde{g}(t, w) \text { where } \widetilde{g}(t, u) \doteq-g(T-t, u),
$$

with initial data

$$
w_{0}(x) \doteq u_{T}(-x)+v^{0}(T) \quad \forall x \in \mathbb{R}
$$


and observe that the function $u(t, x) \doteq w(T-t,-x)$ is a weak distributional solution of $(9)$, which, by (92), (106), satisfies

$$
u(T, \cdot)=u_{T}+E_{T} 0 .
$$

Moreover, note that because of (92), (106) one has $\widetilde{E}_{t} v^{0}(T)=v^{0}(T-t)$, and $\widetilde{E}_{t} w_{0}=v^{0}(T-t)$ for all $x$ with $|x|$ large enough. Hence, with the same arguments as above, by virtue of (12), (102), (106), and because $u_{T} \in \mathcal{A}_{T}$, one deduces that

$$
\begin{array}{lr}
\left\|w(t, \cdot)-\widetilde{E}_{t} v^{0}(T)\right\|_{L^{\infty}} \leq \exp \left(\|\omega\|_{L^{1}(0, t)}\right) \cdot\left\|u_{T}\right\|_{L^{\infty}} \leq \exp \left(\|\omega\|_{L^{1}(0, T)}\right) \cdot h \leq M & \forall t \in[0, T], \\
\left\|w(t, \cdot)-\widetilde{E}_{t} v^{0}(T)\right\|_{L^{1}} \leq \exp \left(\|\omega\|_{L^{1}(0, t)}\right) \cdot\left\|u_{T}\right\|_{L^{1}} \leq \exp \left(\|\omega\|_{L^{1}(0, T)}\right) \cdot L h \leq m & \forall t \in[0, T] .
\end{array}
$$

Recalling the definition (19) of $G_{T}$, from (98), (102) and (108) we derive

$$
\|w(s, \cdot)\|_{L^{\infty}} \leq G_{T}, \quad \forall s \in[0, T] .
$$

Thus, observing that $\widetilde{E}_{T} v_{0}(T)=v_{0}(0)=0$, setting

$$
\begin{aligned}
& \widetilde{L}_{1}(T) \doteq-\sup \left\{x \in \operatorname{Supp}\left(\widetilde{E}_{T} w_{0}\right) \mid w_{0}(-\cdot) \in \mathcal{A}_{T}+v^{0}(T)\right\} \\
& \widetilde{L}_{2}(T) \doteq-\inf \left\{x \in \operatorname{Supp}\left(\widetilde{E}_{T} w_{0}\right) \mid w_{0}(-\cdot) \in \mathcal{A}_{T}+v^{0}(T)\right\},
\end{aligned}
$$

and relying on (102) and (104), we deduce that

$\operatorname{Supp}(w(T,-\cdot)) \subset\left[\widetilde{L}_{1}(T), \widetilde{L}_{2}(T)\right], \quad \widetilde{L}_{2}(T)-\widetilde{L}_{1}(T) \leq 2 \widetilde{L}_{T}+2 T\left\|f^{\prime \prime}\right\|_{L^{\infty}\left(-G_{T}, G_{T}\right)} \cdot \exp \left(\|\omega\|_{L^{1}(0, T)}\right) h \leq 2 L$

Then, setting

$$
\tau_{T} \doteq \widetilde{L}_{1}(T)+L
$$

we find that $\operatorname{Supp}\left(\mathcal{T}_{-\tau_{T}}(w(T,-\cdot))\right)=\operatorname{Supp}\left(\mathcal{T}_{-\tau_{T}}\left(\widetilde{E}_{T} w_{0}(-\cdot)\right)\right) \subset[-L, L]$, which, together with the estimates (108)-(109), yields

$$
\mathcal{T}_{-\tau_{T}}(u(0, \cdot))=\mathcal{T}_{-\tau_{T}}(w(T,-\cdot)) \in \mathcal{C}_{[L, m, M]} .
$$

Therefore, since $w(t, x)$ verifies the upper one-side inequality (48), if we establish the lower bound

$$
w_{x}(t, \cdot) \geq-\widetilde{C}_{t} \text { in the sense of measures, }
$$

for some constant $\widetilde{C}_{t}>0$, and for all $\left.t \in\right] 0, T\left[\right.$, it would follow that $u(t, \cdot) \in \operatorname{Lip}_{l o c}(\mathbb{R})$ for all $t \in(0, T)$, and hence, observing that $\mathcal{T}_{-\tau_{T}}(u(t, \cdot))=E_{t}\left(\mathcal{T}_{-\tau_{T}}(u(0, \cdot))\right)$, we deduce by (107) that

$$
\mathcal{T}_{-\tau_{T}}\left(u_{T}\right) \in E_{T}\left(\mathcal{C}_{[L, m, M]}\right)-E_{T} 0 .
$$

In turn, this relation clearly implies $u_{T} \in \mathcal{T}_{\tau_{T}}\left(E_{T}\left(\mathcal{C}_{[L, m, M]}\right)\right)-E_{T} 0$, proving (105) and hence (103).

Concerning (111), we follow the proof of Propositions 1 and 3 . We introduce $T_{1}$ by (30) and as before define $v$ by $v(t, x) \doteq w_{x}(t, x)$. We get that $v$ is the unique broad solution on $\left[0, T_{1}[\times \mathbb{R}\right.$ of the semilinear equation

$$
v_{t}(t, x)+f^{\prime}(w(t, x)) \cdot v_{x}(t, x)=\widetilde{g}_{u}(t, w(t, x)) \cdot v(t, x)-f^{\prime \prime}(w(t, x)) \cdot v(t, x)^{2} .
$$

We introduce $z(t) \doteq v(t, x(t))$ as the value of $v$ along a characteristic associated to (9) and consider a compact interval $\left[\tau_{1}, \tau_{2}\right] \subset\left[0, T_{1}[\right.$, during which $z(t)<0$, and such that

$$
z\left(\tau_{1}\right) \geq-\left(T\left\|f^{\prime \prime}\right\|_{L^{\infty}\left(-G_{T}, G_{T}\right)} \exp \left(\|\omega\|_{L^{1}(0, T)}\right)\right)^{-1} .
$$

We obtain that

$$
\dot{z}(t) \geq \omega(t) \cdot z(t)-\left\|f^{\prime \prime}\right\|_{L^{\infty}\left(-G_{T}, G_{T}\right)} \cdot z^{2}(t), \quad t \in\left[\tau_{1}, \tau_{2}\right] .
$$

Now defining $z_{1}$ as previously by $z_{1}(t) \doteq \exp \left(-\int_{0}^{t} \omega(s) d s\right) \cdot z(t)$, we deduce

$$
\dot{z}_{1}(t) \geq-\left\|f^{\prime \prime}\right\|_{L^{\infty}\left(-G_{T}, G_{T}\right)} \exp \left(\|\omega\|_{L^{1}(0, T)}\right) z_{1}^{2}, \quad t \in\left[\tau_{1}, \tau_{2}\right],
$$


which yields

$$
z_{1}(t, \cdot) \geq \frac{-\exp \left(\|\omega\|_{L^{1}(0, T)}\right)}{\frac{1}{z_{1}(T)}-t\left\|f^{\prime \prime}\right\|_{L^{\infty}\left(-G_{T}, G_{T}\right)}} .
$$

With (112), this establishes $T_{1}=T$ and (111) with $\widetilde{C}_{t} \doteq\left((T-t)\left\|f^{\prime \prime}\right\|_{L^{\infty}\left(-G_{T}, G_{T}\right)}\right)^{-1}$. This completes the proof of (103).

Since by (102), (104) we have $\widetilde{L}_{T} \geq(3 / 4) L$, relying on (103), observing that

$$
H_{\varepsilon}\left(E_{T}\left(\mathcal{C}_{[L, m, M]}\right) \mid L^{1}(\mathbb{R})\right)=H_{\varepsilon}\left(\mathcal{T}_{\tau_{T}}\left(E_{T}\left(\mathcal{C}_{[L, m, M]}\right)\right)-E_{T} 0 \mid L^{1}(\mathbb{R})\right),
$$

and applying (24), with $b=\left(T\left\|f^{\prime \prime}\right\|_{L^{\infty}\left(-G_{T}, G_{T}\right)} \exp \left(\|\omega\|_{L^{1}(0, T)}\right)\right)^{-1}, L=\widetilde{L}_{T}$, we derive the estimate (18).

\section{References}

[1] Bartlett P. L., Kulkarni S. R., Posner S. E., Covering numbers for real-valued function classes. IEEE Trans. Inform. Theory 43 (1997), no. 5, 1721-1724.

[2] Bressan A., Hyperbolic systems of conservation laws, Oxford Lecture Series in Mathematics and its Applications 20, Oxford University Press, Oxford, 2000.

[3] Dafermos C. M., Generalized characteristics and the structure of solutions of hyperbolic conservation laws. Indiana Univ. Math. J. 26 (1977), no. 6, 1097-1119.

[4] Dafermos C. M., Hyperbolic conservation laws in continuum physics, Grundlehren Math. Wissenschaften Series, Vol. 325. Second Edition. Springer Verlag, 2005.

[5] De Lellis C., Golse F., A Quantitative Compactness Estimate for Scalar Conservation Laws, Comm. Pure Appl. Math. 58 (2005), no. 7, 989-998.

[6] Glimm J., Lax P. D., Decay of solutions of nonlinear hyperbolic conservation laws. Mem. Amer. Math. Soc., 101 (1970).

[7] Goatin P., Gosse L., Decay of positive waves for $n \times n$ hyperbolic systems of balance laws. Proc. Amer. Math. Soc. 132 (2004), no. 6, 1627-1637.

[8] Hoeffding W., Probability inequalities for sums of bounded random variables. J. Amer. Statist. Assoc. 58 (1963), 13-30.

[9] Kružkov S. N., First order quasilinear equations with several independent variables. Mat. Sb. (N.S.) 81 (123) 1970, 228-255. (Russian) English translation in Math. USSR Sbornik Vol. 10 (1970), No. $2,217-243$.

[10] Lax P. D., Weak solutions of nonlinear hyperbolic equations and their numerical computation. Comm. Pure Appl. Math. 7 (1954), 159-193.

[11] Lax P. D., Accuracy and resolution in the computation of solutions of linear and nonlinear equations. Recent advances in numerical analysis (Proc. Sympos., Math. Res. Center, Univ. Wisconsin, Madison, Wis., 1978). Publ. Math. Res. Center Univ. Wisconsin, 107-117. Academic Press, New York, 1978.

[12] Oleinik O. A., Discontinuous solutions of non-linear differential equations. Uspehi Mat. Nauk (N.S.) 12 (1957) no. 3(75), 3-73. (Russian) English translation in Ann. Math. Soc. Trans. Ser. 2 26, 95-172.

[13] Robyr R., SBV regularity of entropy solutions for a class of genuinely nonlinear scalar balance laws with non-convex flux function. J. Hyperbolic Differ. Equ. 5 (2008), no. 2, 449-475. 